\title{
Reaction rate enhancement by surface diffusion of adsorbates
}

\author{
Dong Wang a , Shi-Yuan Gou ${ }^{\mathbf{b}}$ and Daniel Axelrod ${ }^{\mathrm{a}, \mathrm{c}}$ \\ ${ }^{a}$ Biophysics Research Division, ${ }^{b}$ Department of Mechanical Engineering, and ${ }^{c}$ Department of Physics, \\ University of Michigan, Ann Arbor, MI 48109 (USA)
}

(Received 13 August 1991; accepted in revised form 11 February 1992)

\begin{abstract}
Ligands can be captured by a surface target through either direct bulk diffusion or surface diffusion following reversible adsorption to the surface. We have solved a steady state boundary value problem for a perfect sink disk target in the surface, taking into account bulk and surface diffusion coefficients $D$ and $D_{\mathrm{s}}$ and adsorption/desorption kinetic rate constants $k_{\mathrm{a}}$ and $k_{\mathrm{d}}$ at non-target regions. Solutions have been successfully found by numerical computation. The results show that the rate of capture from the surface depends non-linearly on $D_{\mathrm{s}}, D, k_{\mathrm{a}}, k_{\mathrm{d}}$ and geometrical dimensions. In particular, we demonstrate that not only is the non-target region equilibrium constant $K_{\text {eq }}\left(=k_{\mathrm{a}} / k_{\mathrm{d}}\right)$ important in determining the rate of capture from the surface, but so are the kinetic rate constants $k_{\mathrm{a}}$ and $k_{\mathrm{d}}$ separately. In all cases, the surface adsorption/diffusion combination enhances the total tate of capture. The results should be useful for predicting reaction rates of biological membrane bound receptor clusters and substrate-immobilized enzymes.
\end{abstract}

Keywords: Surface diffusion; Membrane receptors; Reaction kinetics; Surface adsorption; Mathematical model; Reduction of dimensionality

\section{Introduction}

Surfaces are involved in many chemical and biological reactions. A surface often has a direct effect on the kinetics and mechanism of the reaction, particularly if one reactant (called the "target" here) is confined to the surface and the other (called the "ligand" here) is dissolved in the bulk. There are two distinct diffusive pathways for the bulk-dissolved ligand to be captured

Correspondence to: Dr. Daniel Axelrod, Biophysics Research Division, University of Michigan, 2200 Bonisteel Blvd., Ann Arbor, MI 48109. Tel. \# 313-764-5280. by the target sink (see Fig. 1): by pure three-dimensional (3D) diffusion to the "specific" target; or by $3 \mathrm{D}$ diffusion to a "non-specific" (i.e., nontarget) region of the surface followed by adsorption and two-dimensional (2D) surface diffusion to the target. A more general intermediate case can occur if the adsorption to the surface is reversible. In principle, the rates of capture from $3 \mathrm{D}$ and from $2 \mathrm{D}$ affect each other, and the sum of the two rates in steady state is always greater than the steady state rate of capture that would have occurred with a non-adsorbing surface.

Adam and Delbrück [1] first introduced the idea of reaction rate enhancement by "reduction of dimensionality" and suggested certain biologi- 
cal systems where it might be operant (e.g., pheromones and olfactory organs in insects). Other possibilities are reviewed by Axelrod [2]. Berg and Purcell [3] did a quantitative study of the $3 \mathrm{D} / 2 \mathrm{D}$ reduction of dimensionality problem for a surface containing discrete perfect sink targets in steady state. They derived a criterion by which the $2 \mathrm{D}$ rate of capture (i.e., capture from the surface via $2 \mathrm{D}$ diffusion) might become important, provided that the target surface concentration is low enough so that the total rate of capture (i.e., capture from both 2D and 3D) is much less than its maximum possible value (defined for a hypothetical cell whose entire surface is a perfect sink). Their criterion asserts that the $2 \mathrm{D}$ rate of capture is proportional only to the equilibrium constant for adsorption to non-target areas $K_{\text {eq }}\left(=k_{\mathrm{a}} / k_{\mathrm{d}}\right)$ and to the surface diffusion coefficient $D_{\mathrm{s}}$, and that it is independent of the bulk diffusion coefficient $D$ or the kinetic rate constants $k_{\mathrm{a}}, k_{\mathrm{d}}$ for non-specific adsorption/desorption. According to their theory, the $2 \mathrm{D}$ rate of capture per target decreases as the surface target concentration decreases. More recently, Otto Berg [4] analytically solved a mathematical model of reversible adsorption and surface diffusion to a single 2D perfect sink target. However, as Berg mentions, that model is most appropriate for the limit where the kinetic adsorption and desorption rate constants approach infinity.
Cukier [5] has presented an elegant approximate mathematical formalism for a somewhat different but related problem, in which the target is a perfect sink circular ring instead of a disk, the adsorption/desorption process is assumed to be saturable and can occur anywhere on the surface (even directly into the central area of the ring), and the bulk concentration is assumed to be constant everywhere. This formalism would be expected to correspond best to the above models where the targets are small compared to the whole surface.

Here, we present a general computational solution of the problem of surface-confined perfect sinks able to receive ligands from either $2 \mathrm{D}$ or 3D. Solutions are found by numerical calculations on a personal computer. Our results show that the $2 \mathrm{D}$ rate of capture per target not only is a function of $K_{\text {eq }}$, but also depends on the adsorption/desorption kinetic rate constants $k_{\mathrm{a}}$ and $k_{\mathrm{d}}$ separately. In addition, this $2 \mathrm{D}$ rate of capture has a non-linear dependence on $D_{\mathrm{s}}$ and it depends upon the value of the $3 \mathrm{D}$ bulk diffusion coefficient. Also, the 2D rate of capture per target increases (rather than decreases as in the Berg and Purcell model) and approaches an asymptote as the surface target concentration decreases. These significant discrepancies between our results and those of Berg and Purcell evidently arise from their more restrictive assump-
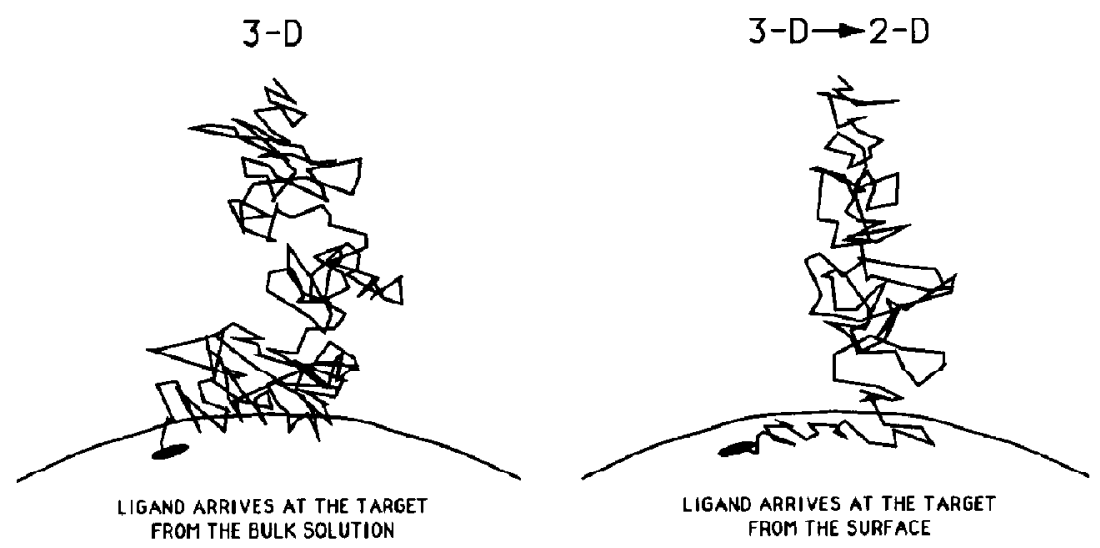

Fig. 1. Two possible pathways for the capture of ligands by the target. 
tions. It will also be shown that our results agree with those from Otto Berg, but only in the limit when $k_{\mathrm{a}}$ and $k_{\mathrm{d}} \rightarrow \infty$.

All of the above models assume that the target is a perfect sink. Several processes in industrial chemistry and cell biology can conceivably be described by a model of $3 \mathrm{D} / 2 \mathrm{D}$ diffusion to a perfect sink. For example, in industrial applications, enzymes immobilized on the surfaces of artificial matrices are used to catalyze the conversion of large quantities of bulk dissolved ligands into useful products [6]. If the rate of conversion of a particular ligand to product is limited only by the time necessary for that ligand to diffuse to an enzyme, then that enzyme can be modeled as a perfect sink for the ligand. Numerous biological cell surface enzymes also can act like immobilized sinks for bulk-dissolved ligands. For example, acetylcholinesterase resides in the basal lamina at the muscle cell surface of a synapse and captures acetylcholine, breaking it into acetate and choline and thereby shortening the time that free acetylcholine is available to interact with the separate acetylcholine receptors. If acetylcholine nonspecifically adsorbs to the cell surface, the breakdown reaction rate will be affected.

Another possible example of a naturally occurring $2 \mathrm{D}$ perfect sink is the general class of membrane transporters. A membrane transporter transfers a specific type of molecule (e.g., sugars, amino acids, nucleotides or some cell metabolites) across cell membranes. If we assume that the transporter functions unidirectionally, and that the time required for transferring is much smaller than the time between arrivals of molecules, then the transporter can be modeled as a perfect sink.

A distinctive feature of a perfect sink is that it creates, and is affected by, a local diffusion depletion zone around itself in steady state. However, many cell surface receptors cannot be modeled as perfect sinks. In some situations, receptors reversibly bind ligands, releasing them unaltered back into solution, and thereby establishing an equilibrium with no concentration gradients. Other receptors bind irreversibly but with a capacity of just one ligand, followed by internalization of the whole complex (often by internaliza- tion at a coated pit), as new receptors are continuously incorporated into the membrane in a steady state process. If these new replacement receptors are incorporated in a spatially random pattern (only an approximation to biological reality), then the fresh unoccupied receptors see a uniform probability density of ligand in their vicinities, and again, no local depletion zones are relevant for calculating rates. (A relevant global deplction zone still develops ncar the surfacc.) However, a pre-existing cluster of many receptors (e.g., for neurotransmitters, hormones, and pheromones) still can be modeled as a perfect sink if the cluster as a whole does not readily become saturated by captured ligands. Certain cell surface receptors are found in clusters before capturing ligands, e.g., acetylcholine receptors in both synapses and in aneural muscle cultures, and nerve growth factor receptors [7].

If the concentration of surface targets is sufficiently high [3], then even rapid surface diffusion of nonspecifically adsorbed ligands may not significantly speed up encounters with targets. McCloskey and Poo [8] have presented a review of selected biological data which suggests that some potential membrane targets do indeed present a sufficiently high concentration so that surface diffusion would not be helpful. However, some other biological membranes may have lower surface target concentrations so that the rate of capture of ligands from the surface could be significant. More experimental data is necessary to determine the surface target concentrations of other systems.

Another possible instance of rate enhancement entails the reduction of dimensionality of diffusion from three to one. One example of a system where this type of rate enhancement might occur is the non-specific binding and possible $1 \mathrm{D}$ diffusion of a repressor protein along a DNA strand before it encounters its specific operator [9].

The results from this theoretical study can be used for answering questions about the surface contribution to the rate of capture in a real system if the key dynamic parameters - the surface diffusion coefficient and the non-specific reversible adsorption/desorption kinetic rate con- 
stants-are known. Fortunately, these parameters often can be calculated from the results of a variety of techniques, notably by total internal reflection/fluorescence recovery after photobleaching (TIR/FRAP) [10-13].

\section{Mathematical model}

We will refer to the immobile 2D-confined enzymes, transporters, receptor clusters, etc. as targets, and the diffusing hormones, agonists, antigens, etc. as ligands. Binding (assumed irreversible) between the two classes is referred to as specific binding. Binding (assumed reversible) of ligands to all other areas of the surface is referred to as non-specific adsorption.

Each target is modeled as a disk sink with radius $R_{1}$ embedded in the surface (see Fig. 2). The boundary for each target is chosen to be a cylinder of radius $R_{2}$ and height $H$, with the target at the center of its bottom surface. $R_{2}$ is determined by the target concentration on the cell surface. For a cell that has many targets uniformly distributed on its surface, each target on the average occupies an area $A_{\mathrm{r}}$ that is equal to the total area divided by the total number of targets. If $A_{\mathrm{r}}$ is flat, it can be modeled as a circle with the target in the center, $R_{2}$ is defined as the radius of that circle.

\subsection{Definitions}

Notation definitions are summarized here and explained later as necessary.

$C$ is the bulk (3D) concentration of ligands (number $/ \mathrm{cm}^{3}$ ) and $C_{\mathrm{s}}$ the surface (2D) concentration of ligands (number $/ \mathrm{cm}^{2}$ ). The non-specific binding reaction is:

$C \underset{k_{\mathrm{d}}}{\stackrel{k_{\mathrm{a}}}{\rightleftharpoons}} C_{\mathrm{s}}$

where $k_{\mathrm{a}}$ is the adsorption rate constant of ligands to non-target areas $(\mathrm{cm} / \mathrm{s}), k_{\mathrm{d}}$ the desorption rate constant of ligands from non-target areas $\left(\mathrm{s}^{-1}\right)$, and $K_{\text {eq }}$ the equilibrium constant for ligand adsorption to non-target areas $(\mathrm{cm})$ equal to $k_{\mathrm{a}} / k_{\mathrm{d}}$. The diffusion coefficients are: $D$ the bulk diffusion coefficient of ligands $\left(\mathrm{cm}^{2} / \mathrm{s}\right)$, and

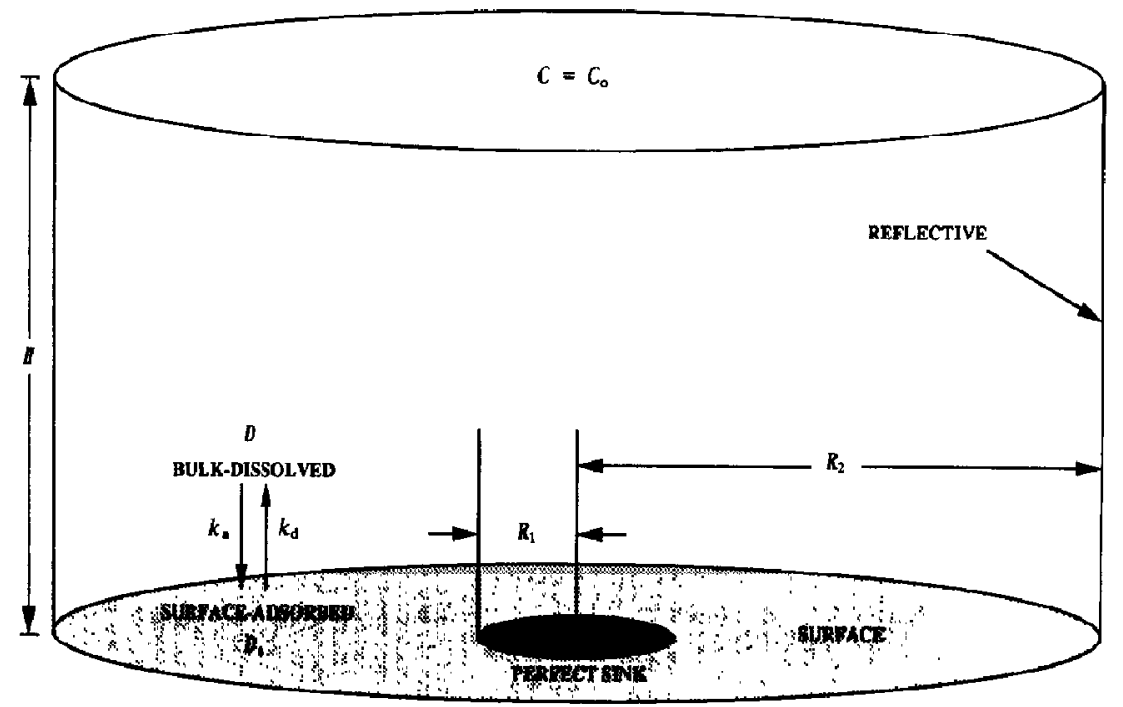

Fig. 2. Geometry and boundary conditions. A disk perfect sink is embedded in the center at the bot tom of the cylinder. 
$D_{\mathrm{s}}$ the surface diffusion coefficient of ligands in nont-arget areas $\left(\mathrm{cm}^{2} / \mathrm{s}\right)$. The geometrical features are: $R_{1}$ the radius of the target $(\mathrm{cm}), R_{2}$ the radius of the cylinder $(\mathrm{cm})$, and $H$ the height of the cylinder $(\mathrm{cm})$. The flux rates per target are: $F_{2 \mathrm{D}}$ the rate of capture of ligands by the target from the (2D) surface (number/s). $F_{3 \mathrm{D}}$ the rate of capture of ligands by the target from the (3D) bulk (number/s), and $F_{\text {tot }}$ the total rate of capture of ligands $\left(F_{2 \mathrm{D}}+F_{3 \mathrm{D}}\right)$ by the target (number $/ \mathrm{s}$ ), respectively. $f_{2 \mathrm{D}}$ is the fraction of the total rate of capture occurring from the $2 \mathrm{D}$ surface, i.e. $F_{2 \mathrm{D}} / F_{\text {tot }} \cdot F_{\max }$ is the maximum possible flux assuming $R_{1}=R_{2}$ (number $/ \mathrm{s}$ ), and $F_{0}$ is the rate of capture assuming $D_{\mathrm{s}}=0$ (number/s). In addition, we will refer to one other rate, defined for a limiting geometry, viz. $F_{0}^{\infty}$ the rate of capture assuming the boundary is set to infinity and $D_{\mathrm{s}}=0$. This special case is called Weber's disk, discussed in Crank [14] where it is shown that:

$F_{0}^{\infty}=4 D R_{1} C_{0}$

\section{$2.23 D$ diffusion equation in steady state}

$$
\nabla^{2} C(r, z)=0
$$

\section{3 $2 D$ diffusion equation in steady state}

For non-targct rcgions of the surface $R_{1}<r<$ $R_{2}$, a source term and a sink term on the right represent non-specific adsorption and desorption, respectively, at the surface:

$$
D_{\mathrm{s}} \nabla^{2} C_{\mathrm{s}}(r)=-k_{\mathrm{a}} C(r, 0)+k_{\mathrm{d}} C_{\mathrm{s}}(r)
$$

\subsection{Boundary conditions}

A finite concentration at a finite height $z=H$ must be maintained for a steady state solution to exist:

$$
C(r, H)=C_{0}
$$

The ligand concentration at the perfect sink target must be zero:

$$
\begin{aligned}
& C\left(r \leqslant R_{1}, 0\right)=0 \\
& C_{\mathrm{s}}\left(r \leqslant R_{1}\right)=0
\end{aligned}
$$

The walls of the cylinder are reflective, equivalent to zero net flow across boundaries of target domains:

$$
\begin{aligned}
& \left.\frac{\partial C(r, z)}{\partial r}\right|_{r=R}=0 \\
& \left.\frac{\partial C_{s}(r)}{\partial r}\right|_{r=R_{2}}=0
\end{aligned}
$$

The bulk concentration gradient in the $z$-direction near the surface is proportional to the net (adsorption minus desorption) flux of ligand adsorbing at the surface for all $R_{1}<r<R_{2}$ :

$$
\left.D \frac{\partial C(r, z)}{\partial z}\right|_{z=0}=k_{\mathrm{a}} C(r, 0)-k_{\mathrm{d}} C_{\mathrm{s}}(r)
$$

It is this rather complicated boundary condition which necessitates a numerical rather than analytical solution to the problem. If, instead of eq. (10), we had $\partial C(r, z) /\left.\partial z\right|_{z=0}=0$ for $R_{1}<r<$ $R_{2}$, then the non-target region could be considered to be a perfect reflector. For the purpose of calculating fluxes in such a case, the condition of perfect reflection can be simulated simply by setting $D_{\mathrm{s}}=0$ in our model, as can be seen by combining eqs. (4) and (10).

Because these boundary conditions all have azimuthal symmetry, $C$ and $C_{\mathrm{s}}$ also have azimuthal symmetry. Hence, $C$ is a function only of $r$ and $z$, and $C_{\mathrm{s}}$ a function only of $r$.

\subsection{Rates of capture per target}

The rates of capture per target region are equal to the integrated diffusive fluxes. A ligand can be captured either through bulk or surface diffusion:

$F_{3 \mathrm{D}}=\left.\int_{0}^{R_{1}} D \frac{\partial C(r, z)}{\partial z}\right|_{z=0} 2 \pi r \mathrm{~d} r$ 
$F_{2 \mathrm{D}}=\left.D_{\mathrm{s}} \frac{\partial C_{\mathrm{s}}(r)}{\partial r}\right|_{r=R_{1}} 2 \pi R_{1}$

$F_{\text {tmax }}$ is defined by assuming the whole bottom area of the cylinder is a perfect sink. It can easily be found by solving the 3D diffusion equation, and the result is:

$F_{\max }=\pi R_{2}^{2} D C_{0} / H$

\subsection{Conversion to dimensionless parameters}

These equations can be made dimensionless in order to highlight the independent variables and to facilitate the analysis of the results. We define the following variables and operators:

$C^{\prime} \equiv C / C_{0}$

$C_{\mathrm{s}}^{\prime} \equiv C_{\mathrm{s}} /\left(D R_{1} C_{0} / D_{\mathrm{s}}\right)$

$r^{\prime} \equiv r / R_{1}$

$z^{\prime} \equiv z / R_{1}$

$\nabla^{\prime 2} \equiv R_{1}^{2} \nabla^{2}$

where the derivatives of $\nabla^{\prime 2}$ are taken with respect to the primed variables $r^{\prime}$ and $z^{\prime}$.

We also define the following ratios of parameters which serve as independent variables:

$a_{1} \equiv R_{2} / R_{1}$

$a_{2} \equiv H / R_{1}$

$a_{3} \equiv k_{\mathrm{a}} R_{1} / D$

$a_{4} \equiv k_{\mathrm{d}} R_{1}^{2} / D_{\mathrm{s}}$

Equations (3)-(10) can now be rewritten in dimensionless form as follows.

3D diffusion equation:

$\nabla^{\prime 2} C^{\prime}=0$

2D diffusion cquation:

For $1<r^{\prime}<a_{1}$,

$\nabla^{\prime 2} C_{s}^{\prime}=-a_{3} C^{\prime}+a_{4} C_{s}^{\prime}$

Boundary conditions:

$C^{\prime}\left(a_{2}\right)=1$

$C^{\prime}\left(r^{\prime} \leq 1,0\right)=0$
$C_{\mathrm{s}}^{\prime}\left(r^{\prime} \leq 1\right)=0$

$\left.\frac{\partial C^{\prime}}{\partial r^{\prime}}\right|_{r^{\prime}=a_{1}}=0$

$\left.\frac{\partial C_{\mathrm{s}}^{\prime}}{\partial r^{\prime}}\right|_{r^{\prime}=a_{1}}=0$

For $1<r^{\prime}<a_{1}$,

$\left.\frac{\partial C^{\prime}}{\partial z^{\prime}}\right|_{z^{\prime}=0}=a_{3} C^{\prime}-a_{4} C_{s}^{\prime}$

Rates of capture per target:

$F_{3 \mathrm{D}}=\left.D C_{0} R_{1} \int_{0}^{1} \frac{\partial C^{\prime}}{\partial z^{\prime}}\right|_{z^{\prime}-0} 2 \pi r^{\prime} \mathrm{d} r^{\prime}$

$F_{2 \mathrm{D}}=\left.D C_{0} R_{1} 2 \pi \frac{\partial C_{\mathrm{s}}^{\prime}}{\partial \mathrm{r}^{\prime}}\right|_{r^{\prime}=1}$

Although there are eight physical parameters $\left(R_{1}, R_{2}, H, D, D_{\mathrm{s}}, k_{\mathrm{a}}, k_{\mathrm{d}}\right.$, and $\left.C_{0}\right)$, there are only four independent variables $\left(a_{1}, a_{2}, a_{3}\right.$, and $a_{4}$ ). This means that for the same set of independent variables but different physical parameters, the solutions for $C$ and $C_{\mathrm{s}}$ each change only by a multiplicative factor (although possibly a different factor for $C$ and $C_{\mathrm{s}}$ ). Fraction $f_{2 \mathrm{D}}$ remains constant, however; it is only a function of the independent variables.

Variables $a_{1}$ and $a_{2}$ are purely geometrical; they are the radius and height, respectively, of the cylinder normalized with respect to the radius of the sink. All of other physical parameters are incorporated into $a_{3}$ and $a_{4}$, the normalized adsorption and desorption rate constants, respectively. In particular, changing only $k_{\mathrm{a}}$ and $D$ by the same factor will not change $a_{3}$, and thereby will not change the solutions for $C^{\prime}$ and $C_{s}^{\prime}$. Likewise, changing only $k_{\mathrm{d}}$ and $D_{\mathrm{s}}$ by the same factor will change neither $a_{4}$ nor the solutions for $C^{\prime}$ and $C_{\mathrm{s}}^{\prime}$.

\section{Previous results}

Berg and Purcell [3] studied a similar problem for a plane by a somewhat different approach, denoted here as the "BP model". Under the limit 
that $F_{2 \mathrm{D}} \ll F_{\max }$ and $F_{3 \mathrm{D}} \ll F_{\max }$, their results for rates of capture per target are, in our notation:

$F_{2 \mathrm{D}}^{\mathrm{BP}}=\frac{2 \pi D_{\mathrm{s}} K_{\mathrm{eq}} C_{0}}{\ln \left(4 R_{2} / 3 R_{1}\right)}$

$F_{3 D}^{\mathrm{BP}}=4 D R_{1} C_{0}$

They therefore concluded that the 2D capture rate will dominate $\left(F_{2 \mathrm{D}}>F_{3 \mathrm{D}}\right)$ if

$\frac{\pi D_{\mathrm{s}} K_{\mathrm{eq}}}{2 D R_{1}}>\ln \left(4 R_{2} / 3 R_{1}\right)$

Equation (33) was derived for a planar surface by calculating the distribution of times-to-capture $f\left(t_{\mathrm{c}}\right)$ assuming a uniform surface concentration of ligands, and then averaging the times-to-capture $t_{c}$ over all possible positions. Under this assumption, $f\left(t_{c}\right)$ is exponential:

$f\left(t_{c}\right)=\frac{1}{\left\langle t_{c}\right\rangle} \mathrm{e}^{-t_{c} /\left\langle t_{c}\right\rangle}$

and the mean time-to-capture (in our notation) is given by

$\left\langle t_{\mathrm{c}}\right\rangle=\frac{R_{2}^{2}}{2 D_{\mathrm{s}}}\left[\ln \left(\frac{R_{2}}{R_{1}}\right)-\frac{3}{4}\right]$

Berg and Purcell assume that the reciprocal of $\left\langle t_{c}\right\rangle$ is proportional to the $2 \mathrm{D}$ flux rate. This is questionable, particularly considering that the $2 \mathrm{D}$ flux rate then approaches zero as $R_{2}$ approaches infinity. Moreover, if the target is a perfect sink, the presence of local depletion zones invalidate eqs. (33-37) for the following two reasons:

(a) In deriving eqs. (33), (36), and (37), it was assumed that the bulk concentration was uniform (and set equal to $C_{0}$ ) and that the bulk and the surface are in equilibrium. These assumptions lead to a uniform surface concentration of ligands and to $F_{2 \mathrm{D}}^{\mathrm{BP}}$ (eq. 33) independent of the geometry in the $z$-direction (i.e., the height of the cylinder). However, a perfecl sink largel will always generate a ligand depletion zone in both the surface and bulk concentrations near the target. Therefore, those ligands near the target-those with the smallest times-to-capture-should be less weighted during averaging in the calculation of $f\left(t_{\mathrm{c}}\right)$. This means that $f\left(t_{\mathrm{c}}\right)$ of eq. (36) overestimates the shorter times, and the correct mean time-to-capture $\left\langle t_{c}\right\rangle$ should be greater than that given in eq. (37). The depth, shape, and size of the depletion zone on the surface should depend on the bulk and surface diffusion coefficients and on the surface kinetic rate constants, since these parameters affect the replenishment rate of a surface depletion zone. Thus, both $f\left(t_{\mathrm{c}}\right)$ and $\left\langle t_{\mathrm{c}}\right\rangle$, and thereby $F_{2 \mathrm{D}}$, should depend on $D, k_{\mathrm{a}}$, and $k_{\mathrm{d}}$ as well as on $D_{\mathrm{s}}, R_{1}$, and $R_{2}$.

(b) When deriving eq. (34), a sphere was used with small circular sinks sparsely and uniformly distributed on its surface. The surface outside the sinks was assumed to be perfectly reflecting, i.e., the $2 \mathrm{D}$ rate of capture is zero. The $F_{3 \mathrm{D}}^{\mathrm{BP}}$ for each sink in the BP model is the same as that the capture rate $F_{0}^{\infty}$ for the case of the Weber's disk (eq. 2), in which a single disk sink is embedded in an infinite reflecting surface with the concentration at infinity held constant. However, when the $2 \mathrm{D}$ route does make some contribution to the rate of capture, the surface cannot be considered to be a perfect reflector. In fact, the deplction zone on the surface will inevitably affect the shape and depth of the depletion zone in the bulk and thereby alter the $3 \mathrm{D}$ rate of capture.

In view of above two considerations, the criterion for dominance of the $2 \mathrm{D}$ rate over the actual $3 \mathrm{D}$ rate will be somewhat different in general from eq. (35).

Otto Berg [4] proposed a model (denoted here as the "OB model") similar to the BP model and our model, but with some important differences. In the OB model, the boundary is set at infinity with the concentration held constant. Since only one target sink on the infinite planar surface is modeled, the dependence of the rate of capture on the surface target density can not be analyzed through this model. Also, the target itself is considered to have the same association rate constant from the bulk as does the rest of the surface; i.e., eq. (30) is valid over the whole surface $0<r^{\prime}<a_{1}$, not just $1<r^{\prime}<a_{1}$. As discussed by the author, this extended boundary condition may not be appropriate in general, but as $k_{\mathrm{a}}$ (and also $k_{\mathrm{d}}$ for a constant $K_{\mathrm{eq}}$ ) approaches infinity, the reversible kinetic behavior of the target itself 
should not matter. At this limit, both OB model and ours should agree if the boundary in our model is positioned far out enough. Results from the OB model in our notation are:

$$
f_{2 \mathrm{D}}^{\mathrm{OB}}=\frac{2\left(\frac{a_{3}}{a_{4}}\right) \int_{0}^{\infty} \frac{J_{1}^{2}(x)\left(1+a_{3}^{-1} x\right)}{1+\left(a_{3} / a_{4}\right) x+a_{4} x^{2}} \mathrm{~d} x}{1-\int_{0}^{\infty} \frac{J_{1}(x) J_{2}(x)}{1+\left(a_{3} / a_{4}\right) x+a_{4} x^{2}} \mathrm{~d} x}
$$

and

$$
\begin{aligned}
& F_{\mathrm{tot}}^{\mathrm{OB}} \\
& \quad=\pi D R_{1} C_{0} /\left[2 \int_{0}^{\infty} \frac{\left[J_{1}(x) / x\right]^{2}\left(1+a_{3}^{-1} x\right)}{1+\left(a_{3} / a_{4}\right) x+a_{4} x^{2}} \mathrm{~d} x\right. \\
& \left.\quad-f_{2 \mathrm{D}}^{\mathrm{OB}} \int_{0}^{\infty} \frac{\left[J_{1}(x) / x\right] J_{2}(x)\left(1+a_{3}^{-1} x\right)}{1+\left(a_{3} / a_{4}\right) x+a_{4} x^{2}} \mathrm{~d} x\right]
\end{aligned}
$$

\section{Computer-generated solution}

Finite difference methods [15] were used to numerically solve the boundary value problem for $C$ and $C_{\mathrm{s}}$ posed in eqs. (23)-(30). Since the expected depletion zone is on the order of the size of the sink in the bulk, the area near the sink should be the most heavily sampled to retain accuracy while reducing the computational time. A non-uniform grid derived from a coordinate transformation [16] was employed in order to sample points more densely near the edge of the target on $r$-coordinate and near the target on the $z$-coordinate. All calculations were performed on a 486-based 33 Mhz MS-DOS personal computer using MicroSoft ForTRAN 5.0-compiled programs. For each set of parameters, the computer required about 2 to 15 minutes to obtain the solutions.

Solutions for concentrations and fluxes of this boundary value problem are functions of the four independent variables $a_{i}$. Plotting the complete results would thereby require a five dimensional graph. Therefore, we present results here based on a rather restricted range of parameters that nonetheless can answer specific questions and be compared with the results of other models. But the programs can be used to find solutions over a wider range of parameters and are available upon request.

\subsection{Verification}

To verify the validity of our geometry, boundary conditions, and computational procedures, we compare some predictions of our model with those of BP model in cases where both models are applicable. First, there is the question of geometry. Most biological cells are neither spherical nor flat, nor are they bathed in an infinite extent of soluble ligand. Rather, the reservoir of ligand is likely to be a confined space between two cell surfaces that curve while maintaining some separation distance (e.g., pre- and post-synaptic membranes). One of the membranes is often a source of ligand while the other contains the targets. The BP model assumes a ligand bath of infinite extent when calculating $F_{3 D}$; our geomctry specifies a finite depth of solution $H$ between the source membrane of ligand and the target membrane. The total capture rate in our model is clearly a function of $H$ for a fixed ligand concentration at $z=H$, but one might expect that the relative contribution of 2D and 3D diffusion would not be a strong function of $H$. Nevertheless, there is some range of $H$ in a our model for which the absolute rate of $3 \mathrm{D}$ capture (in the absence of $2 \mathrm{D}$ diffusion) should agree with a spherical model. As discussed previously, the BP model gives $F_{3 \mathrm{D}}^{\mathrm{BP}} / F_{0}^{\infty}=1$ (see eqs. 2 and 34 ), which is derived assuming that the target area is a very small fraction of the total surface area. In that same range (large $a_{1}$ in our notation), our model also agrees with the Weber disk and BP results, as long as the height and radius of the cylinder are kept on the same order (see Fig. 3).

Berg and Purcell [3] showed that 3D capture by itself can be remarkably efficient on a sphere, even when the targets occupy only a small fraction of a spherical surface area. Furthermore, 2D diffusion can increase $F_{\text {tot }}$ significantly only when the actual rate of capture by $3 D$ is much less than 
the maximum possible rate of $3 \mathrm{D}$ capture $F_{\max }$ as calculated by assuming that the entire surface is a sink. To check this effect in our model, the 3D flux was calculated for cases in which only 3D diffusion exists; i.e., when the surface outside the target is perfectly reflecting. Figure 4 shows the results of this calculation. The fraction $F_{0} / F_{\max }$ (the "3D capture efficiency", defined by eqs. 11 and 13) monotonically decreases from unit as $R_{2}$ increases and reaches 0.5 at some value of $a_{1} \equiv$ $R_{2} / R_{1}$ which depends on the relative height parameter $a_{2}$. In qualitative agreement with Berg and Purcell, if the targets occupy as low as $1 \%$ of the total surface area (in the case of $a_{2}=100$ ), the 3D capture efficiency will still be well over $50 \%$. In real biological systems, specific cell surface targets may occupy even far less of the surface area than $1 \%$. Figure 4 also shows that pure 3D capture becomes more efficient as the relative height of the bulk solution $a_{2}$ increases. This also corresponds to the Berg and Purcell spherical geometry result that $3 \mathrm{D}$ capture becomes more efficient as the radius of the sphere increases relative to the target size $R_{1}$. Unless otherwise indicated, we use $a_{1} \equiv R_{2} / R_{1}=100$ and $a_{2} \equiv H / R_{1}=100$ in this paper. With that geometry, the $3 \mathrm{D}$ capture efficiency is much less than unity and therefore 2D surface diffusion has a chance to enhance $F_{\text {tot }}$.

Figures 5(a-d) explore how the rates of capture per target vary with the surface concentration of targets, for a particular set of diffusion coefficients, kinetic rate constants and target size. These fluxes are plotted as functions of $a_{1}$ (the normalized intertarget spacing) for a set of $a_{2}$ (normalized cylinder height) values. These results show that both the $3 \mathrm{D}$ and the $2 \mathrm{D}$ rates of capture per target increase to a limiting value as the intertarget spacing increases. This behavior is expected: as the surface target concentration becomes less, competition between targets is reduced. Figure 5(d) shows that increasing intertar-

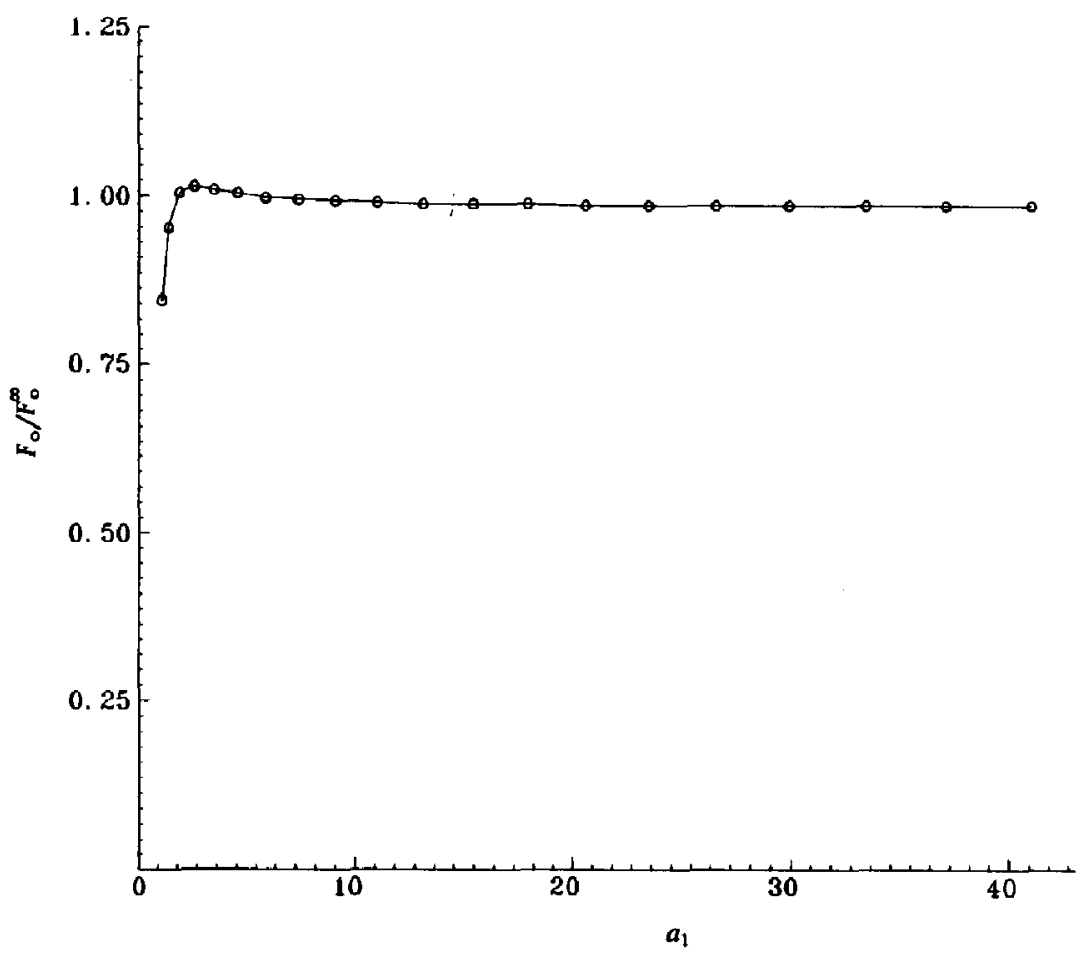

Fig. 3. The effect of geometry on the 3D flux $F_{0}$ (assuming reflecting surface outside the receptor, equivalent to setting $D_{\mathrm{s}}=0$ ), normalized with respect to $F_{0}^{\infty}$, the corresponding $3 \mathrm{D}$ flux except with the $R_{2}$ and $H$ boundaries set at infinity (Weber's disk). Parameters used: $a_{1}=a_{2}, a_{3}=0$, and $a_{4}=0$. After $a_{1}=40, F_{0} / F_{0}^{\infty}$ is essentially unity. 
get spacing increases the 2D fraction of the total rate of capture. Evidently, 2D capture rates suffer more from target crowding than do 3D capture rates. As cylinder height $a_{2}$ increases, both $F_{2 \mathrm{D}}$ and $F_{3 \mathrm{D}}$ (therefore $F_{\mathrm{tuI}}$ ) decrease, but the 2D fraction $f_{2 \mathrm{D}}$ does not seem to vary strongly with the height of the cylinder.

\subsection{Dependence on surface diffusion and kinetic rate parameters}

The effect of surface diffusion on the rates of capture is demonstrated in Figs. 6(a-d). Each rate is normalized to a constant value for $F_{0}$, which is the rate of capture where $D_{\mathrm{s}}=0$ so that reversible $2 \mathrm{D}$ adsorption has no effect on the capture rates. The abscissa in Fig. $6, \log a_{4}^{-1}$, increases linearly with $\log D_{\mathrm{s}}$. Increasing $D_{\mathrm{s}}$ increases $F_{2 \mathrm{D}}, F_{\text {tot }}$ and $f_{2 \mathrm{D}}$ as expected, but decreases $F_{3 \mathrm{D}}$. This is because the very success of 2D capture depletes the surface concentration around the target and, through reversible exchange, thereby depletes the local bulk concentration as well. The depletion of bulk concentration then decreases the 3D rate of capture. This effect is magnified as $a_{3}$ (proportional to the surface adsorption kinetic rate constant) increases. A larger surface adsorption rate replenishes the surface more quickly and therefore enhances the 2D capture rate. But at the same time, it depletes the bulk more severely so that $3 \mathrm{D}$ rate of capture decreases even farther.

Figs. $7(\mathrm{a}-\mathrm{d})$ explore how $F_{2 \mathrm{D}}, F_{3 \mathrm{D}}, F_{\mathrm{tot}}$, and $f_{2 \mathrm{D}}$ vary with the kinetic rate constants for a particular geometry. These capture rates are plotted as functions of $a_{3}$ (normalized adsorption rate) for a set of $a_{4}$ (normalized desorption rate) values. $F_{2 \mathrm{D}}, F_{\mathrm{tot}}$ and $f_{2 \mathrm{D}}$ all increase as $a_{3}$ increases, but $F_{3 \mathrm{D}}$ decreases due to the depletion of the bulk concentration by the surface. The larger the normalized desorption rate $a_{4}$, the larger must be the normalized adsorption rate $a_{3}$ for these effects to occur.

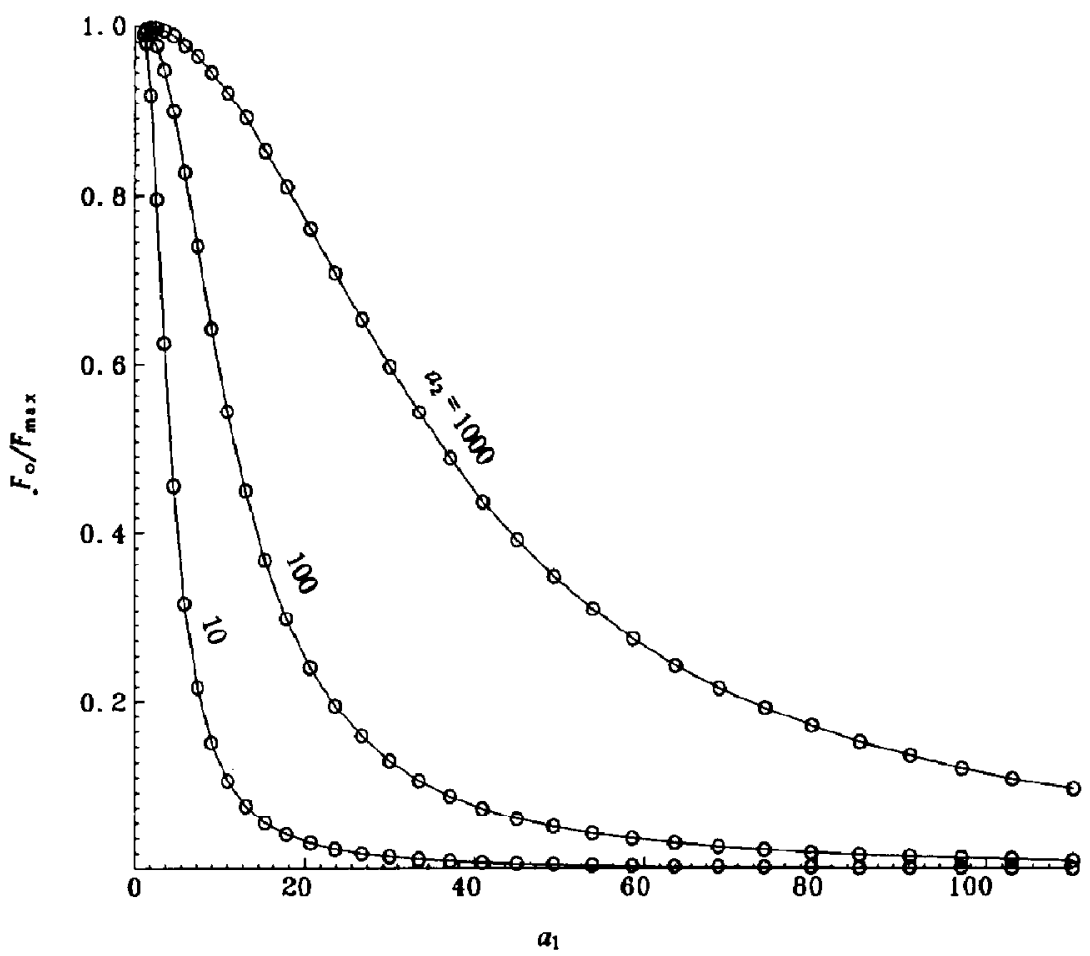

Fig. 4. The effect of surface receptor concentration on the 3D flux for three different relative heights $a_{2}$. Parameters used: $a_{2}=10$, 100 , or 1000 ; and $a_{3}=a_{4}=0$. 

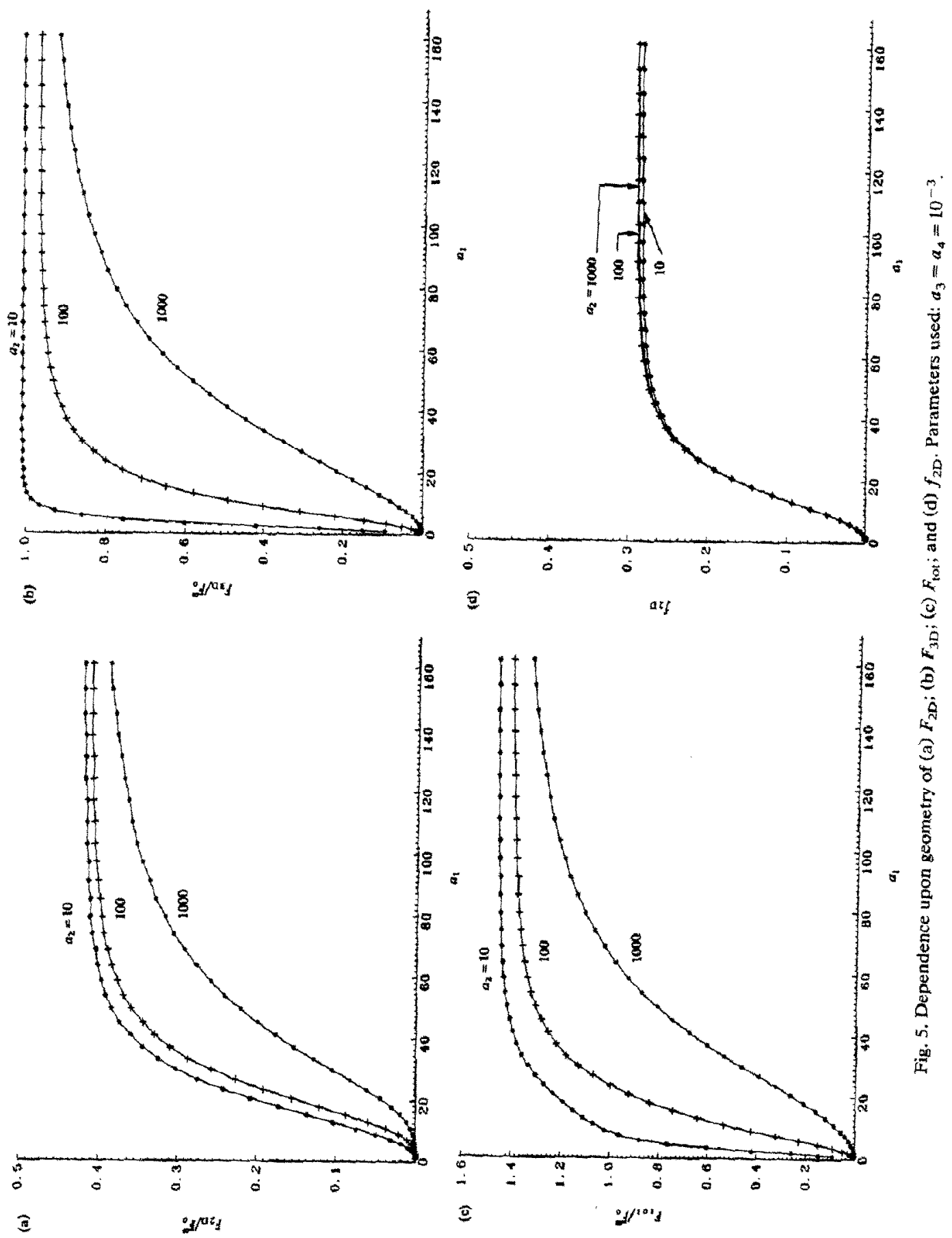


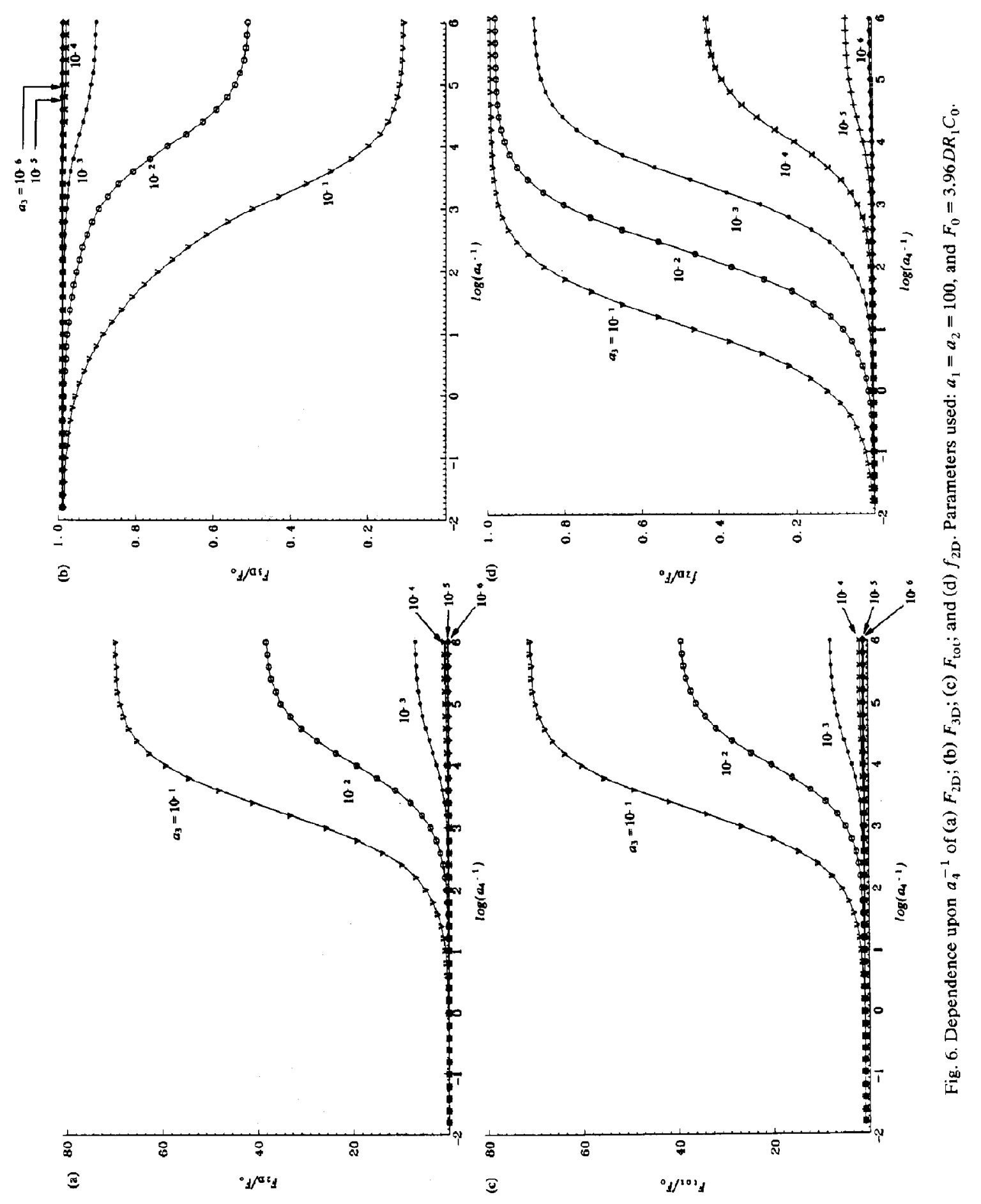



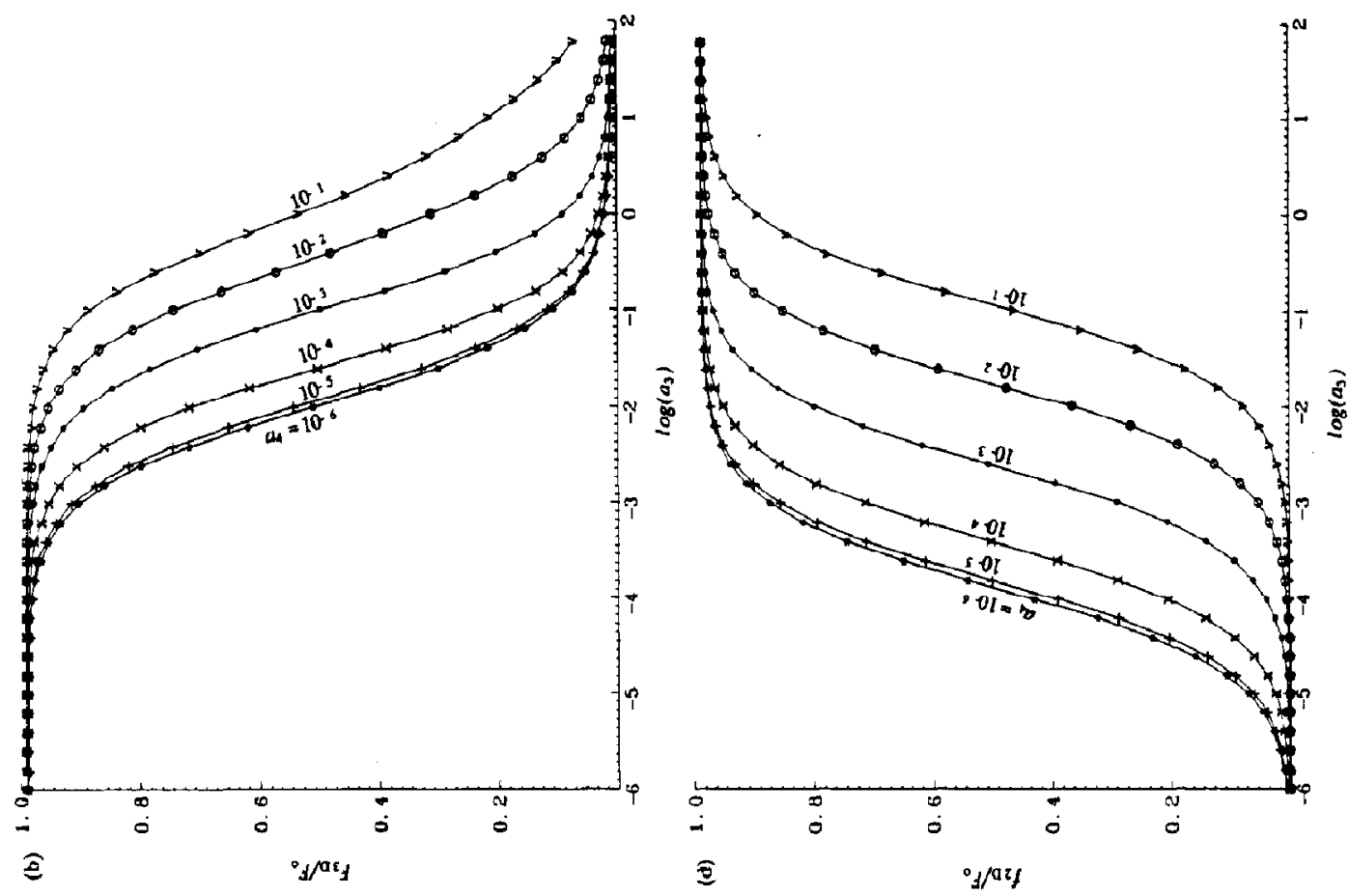

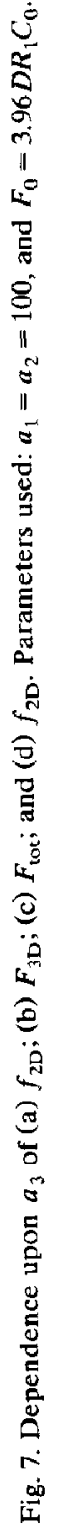
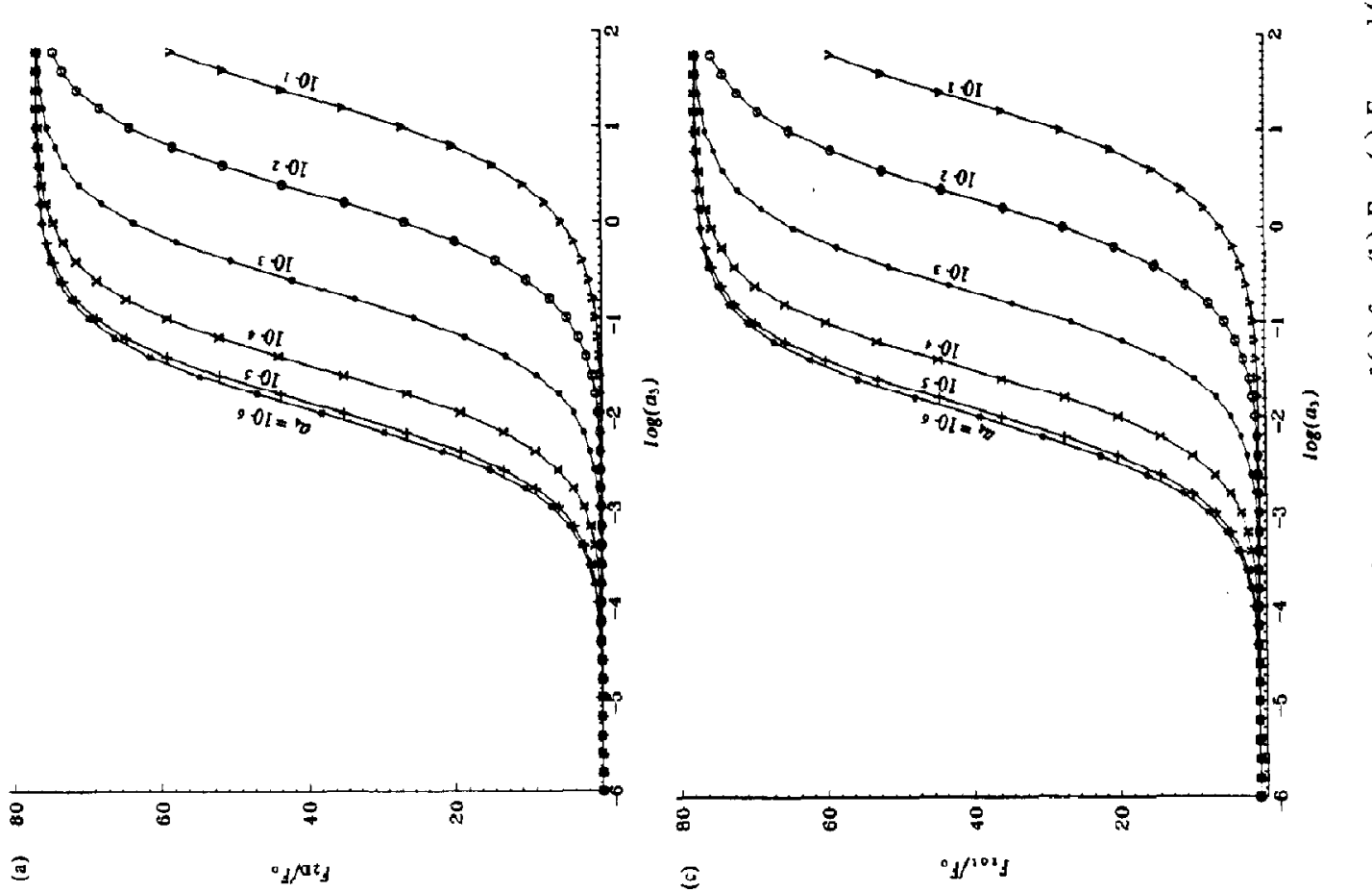


\subsection{Comparison with earlier models}

The results for $F_{2 \mathrm{D}}$ and $F_{3 \mathrm{D}}$ in our model differ from those of Berg and Purcell [3] (eqs. 33 and 34 ) in several respects. For the BP model, eq. (33) states that if $F_{2 \mathrm{D}}$ and $F_{3 \mathrm{D}}$ are $\ll F_{\max }$, then: (1) $F_{2 \mathrm{D}}$ is proportional to $K_{\mathrm{eq}}$, i.e., it does not directly depend on $k_{\mathrm{a}}$ and $k_{\mathrm{d}}$ separately but only on their ratio; (2) $F_{2 \mathrm{D}}$ is proportional to $D_{\mathrm{s}}$; (3) $F_{2 \mathrm{D}}$ does not depend on $D$; and (4) $F_{2 \mathrm{D}}$ decreases as $R_{2}$ increases (as surface target density decreases).

To compare the BP results with our more general results as depicted in Figs. 5, 6 and 7, we must identify where $F_{2 \mathrm{D}}$ and $F_{3 \mathrm{D}}$ are much smaller than $F_{\max }$. For the same set of dynamic parametcrs, these assumptions require a low surface target concentration, i.e., a large $a_{1}$ in Figs. 5(a) and (b). For Figs. 6 and 7, note that our $F_{0} / F_{\max }=0.0125$, so that the assumption of $F_{3 \mathrm{D}}$ $\ll F_{\max }$ is valid throughout Figs. 6(b) and 7 (b). The assumption of $F_{2 \mathrm{D}} \ll F_{\max }$ corresponds to the left portion of each curve in Figs. 6(a) and 7 (a). At regions where these two assumptions are both valid, none of the above four conclusions flowing from the BP model is consistent with our results:

(1) $F_{2 \mathrm{D}}$ in Fig. 7(a) does increases as $a_{3}$ increases, but is not linearly proportional to $a_{3}$ for a constant $a_{4}$. Therefore, $F_{2 \mathrm{D}}$ is clearly not linearly proportional to $K_{\text {eq }}$.

(2) As shown in Fig. 6(a), $F_{2 \mathrm{D}}$ increases with increasing $a_{4}^{-1}$ non-linearly. Since $a_{4}^{-1} \propto D_{\mathrm{s}}$, $F_{2 \mathrm{D}}$ therefore increases with $D_{\mathrm{s}}$ non-linearly.

(3) Because $F_{2 \mathrm{D}} / F_{0}$ depends non-linearly on $a_{3}$ as seen in Fig. 7(a) and $a_{3} \propto D^{-1}, F_{2 \mathrm{D}} / F_{0}$ is a non-linear function of $D^{-1}$. However, since $F_{0}$ must be linearly proportional to $D$, then $F_{2 \mathrm{D}}$ is not independent of $D$.

(4) As shown in Fig. 5(a), $F_{2 \mathrm{D}}$ increases with $a_{1}$, and thus increases with $R_{2}$. This shows that as surface target density decreases, $F_{2 \mathrm{D}}$ increases and reaches an asymptote.

Our results show that $F_{2 \mathrm{D}}$ not only depends on $K_{\mathrm{eq}}$ non-linearly but also varies with $k_{\mathrm{d}}$ (and therefore also $k_{\mathrm{a}}$ ) at any constant $K_{\text {eq }}$. Figures $8(\mathrm{a}-\mathrm{d})$ investigate the dependence of $F_{2 \mathrm{D}}, F_{3 \mathrm{D}}$, $F_{\text {tot }}$, and $f_{2 \mathrm{D}}$ upon $k_{\mathrm{d}}$ for the same $K_{\text {eq }}$, for a set of various $D_{\mathrm{s}}$ values. Other parameters, such as $R_{1}, R_{2}, H, D$ and $C_{0}$, are kept constant for these calculations. The region of $k_{\mathrm{d}}$ plotted in these figures satisfies the condition that $F_{2 \mathrm{D}}$ and $F_{3 \mathrm{D}}$ are much smaller than $F_{\max }$ so that the results can be compared with the BP model. Figure 8 shows that $F_{2 \mathrm{D}}, F_{\text {tot }}$, and $f_{2 \mathrm{D}}$ all increase as $k_{\mathrm{d}}$ increases, and the increase is more dramatic as $D_{\mathrm{s}}$ increases. On the other hand, $F_{3 \mathrm{D}}$ tends to decrease with increasing $k_{\mathrm{d}}$, and increasing $D_{\mathrm{s}}$ enhances the decrease.

The physical origin of the dependence of the rates of capture on the kinetic rate constants and not just the equilibrium constant can be understood by considering the depletion zones both in the bulk and on the surface. Figures $9(\mathrm{a})$ and (b) depict these deletion zones for the two different sets of kinetic rate constants: $k_{\mathrm{a}}$ and $k_{\mathrm{d}}$ fast, and $k_{\mathrm{a}}$ and $k_{\mathrm{d}}$ slow, but for the same equilibrium constant $K_{\text {eq }}$ and the same surface diffusion coefficient $D_{s}$. Faster kinetic rates replenish the surface adsorbed ligand more quickly and increase the $2 \mathrm{D}$ rate of capture while diminishing the width of the surface depletion zone. However, faster replenishment of the surface also depletes the bulk ligand more rapidly so that the bulk has a larger depletion zone. The net effect then is to increase $F_{2 \mathrm{D}}, F_{\text {tot }}$, and $f_{2 \mathrm{D}}$, but to decrease $F_{3 \mathrm{D}}$.

Figures 10(a) and (b) depict the depletion zones for two different surface diffusion coefficients but the same set of kinetic rate constants. Increasing $D_{\mathrm{s}}$ increases the $2 \mathrm{D}$ rate of capture and gives rise to a wider depletion zone on the surface as more distant adsorbed ligands are likely to encounter the target. But this wider surface depletion zone depletes the bulk ligand more rapidly, and thereby causes the bulk to have a larger depletion zone as well. The 3D capture rate thereby decreases.

Our results also can be compared with those of OB model by plotting the results for $F_{\text {tot }} / F_{0}^{\infty}$ (Fig. $11 \mathrm{a}$ ) and $f_{2 \mathrm{D}}$ (Fig. 11b) vs. $a_{3} / a_{4}$ (the normalized adsorption/desorption equilibrium constant) from both models. A large boundary is chosen in order to simulate the infinity boundary in the $O B$ model. A corresponding pair of graphs (Figs. 12a and b) displays the same range of equilibrium constants but faster kinetic rates. Comparison of 

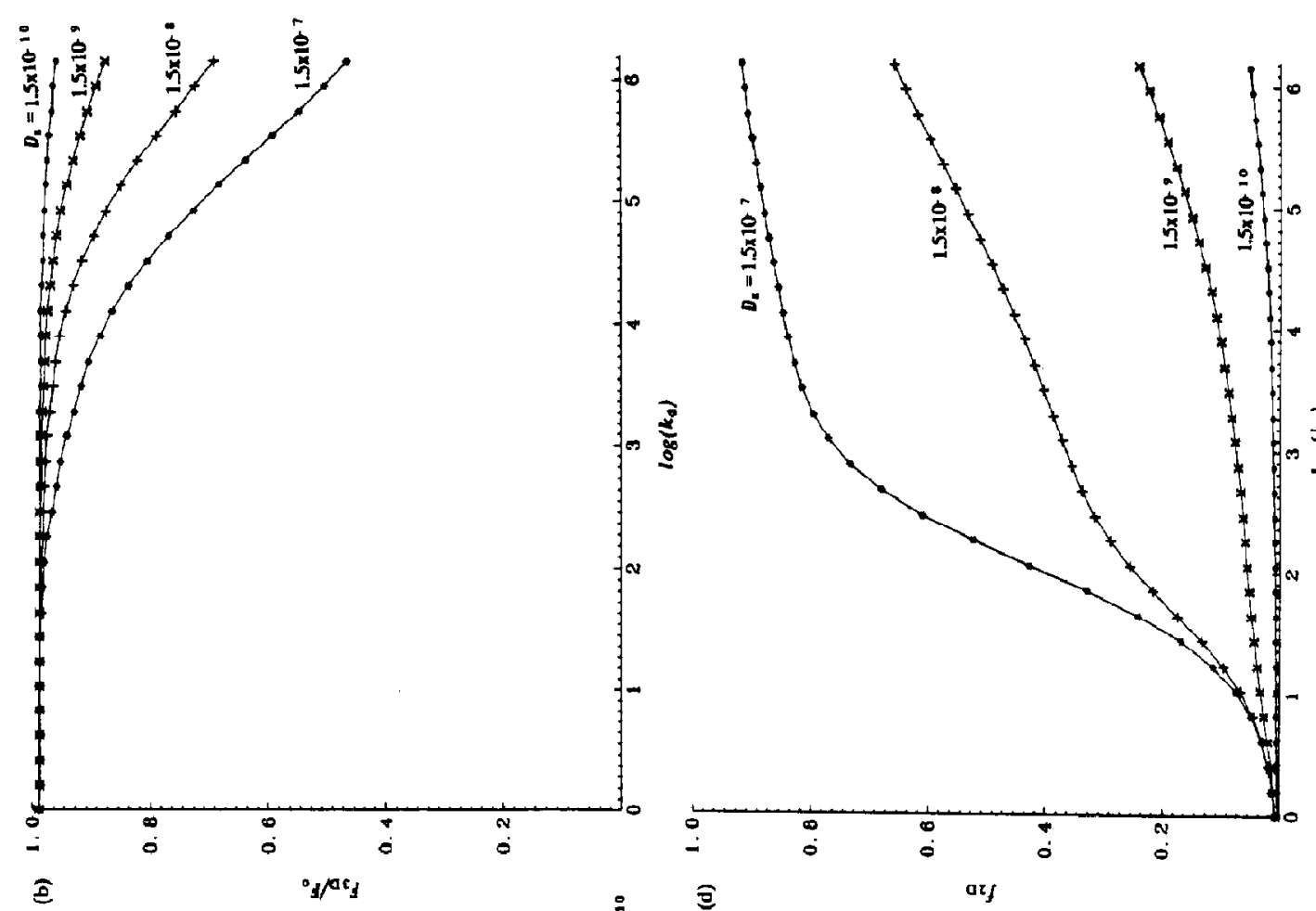

䎏

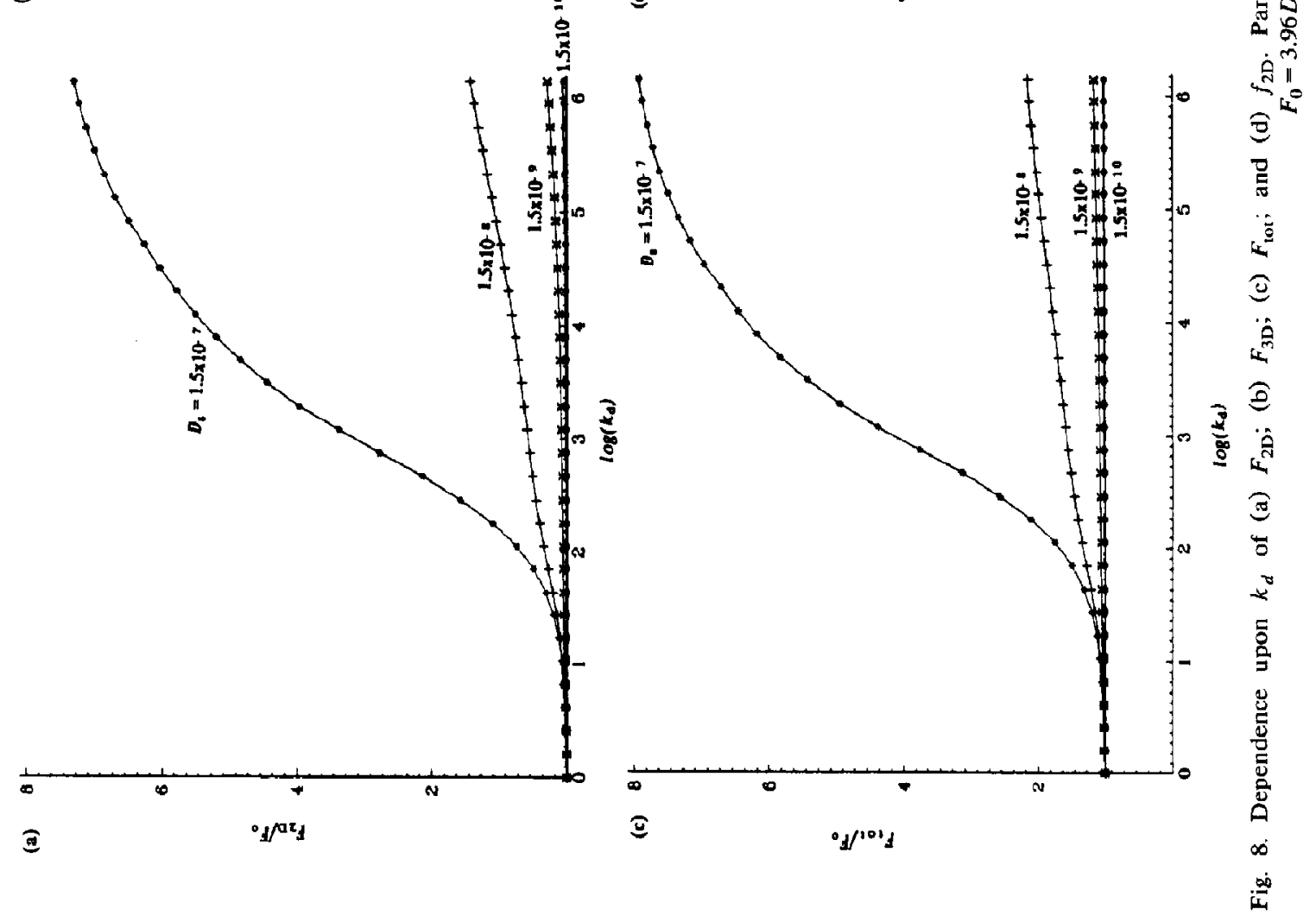



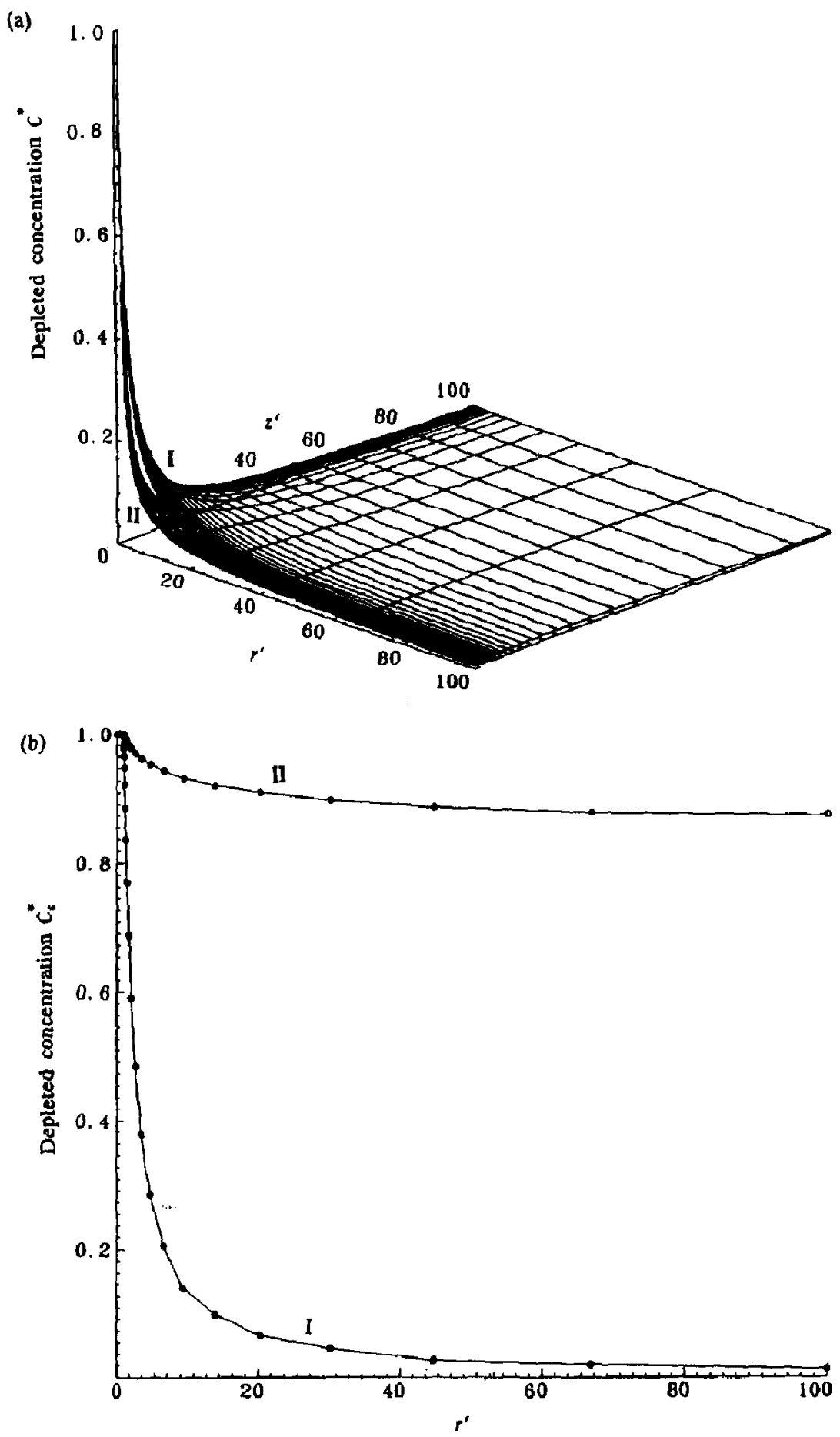

Fig. 9. (a) Effect of kinetic rate constants on bulk depletion zones. The ordinate axis is the depleted concentration defined as $C^{*}=1-C / C_{0}$. (b) Effect of kinetic rate constants on surface depletion zones. The ordinate axis is the depleted concentration defined as $C_{s}^{*}=1-C_{s} / C_{0} K_{\text {eq. }}$. Parameters used: $a_{1}=a_{2}=100, D=1.5 \times 10^{-6}, K_{\mathrm{eq}}=1.5 \times 10^{-5}, D_{\mathrm{s}}=1.58 \times 10^{-8}, R_{1}=10^{-7}$. Curve I: $k_{\mathrm{d}}=10^{6}$, and curve II: $k_{\mathrm{d}}=10$. 

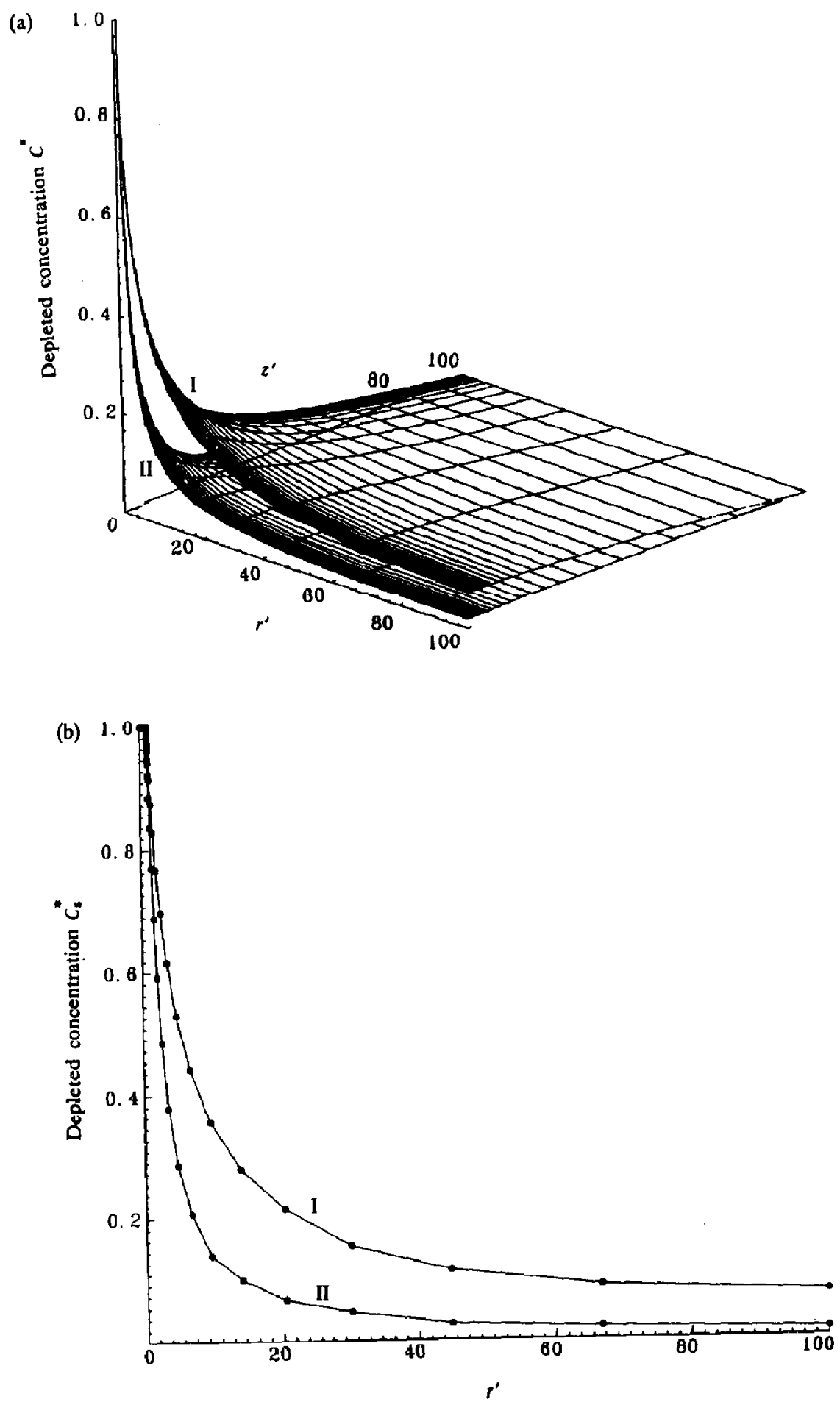

Fig. 10. Effect of surface diffusion on (a) bulk depletion zones, and (b) surface depletion zones. $C^{*}$ and $C_{\mathrm{s}}^{*}$ ordinates are defined as in Fig. 9. Parameters used: $a_{1}=a_{2}=100, D=1.5 \times 10^{-6}, K_{\mathrm{eq}}=1.5 \times 10^{-5}, k_{\mathrm{d}}=10^{6}, R_{1}=10^{-7}$. Curve I: $D_{\mathrm{s}}=1.5 \times 10^{-7}$, and curve II: $D_{\mathrm{s}}=1.5 \times 10^{-8}$. 


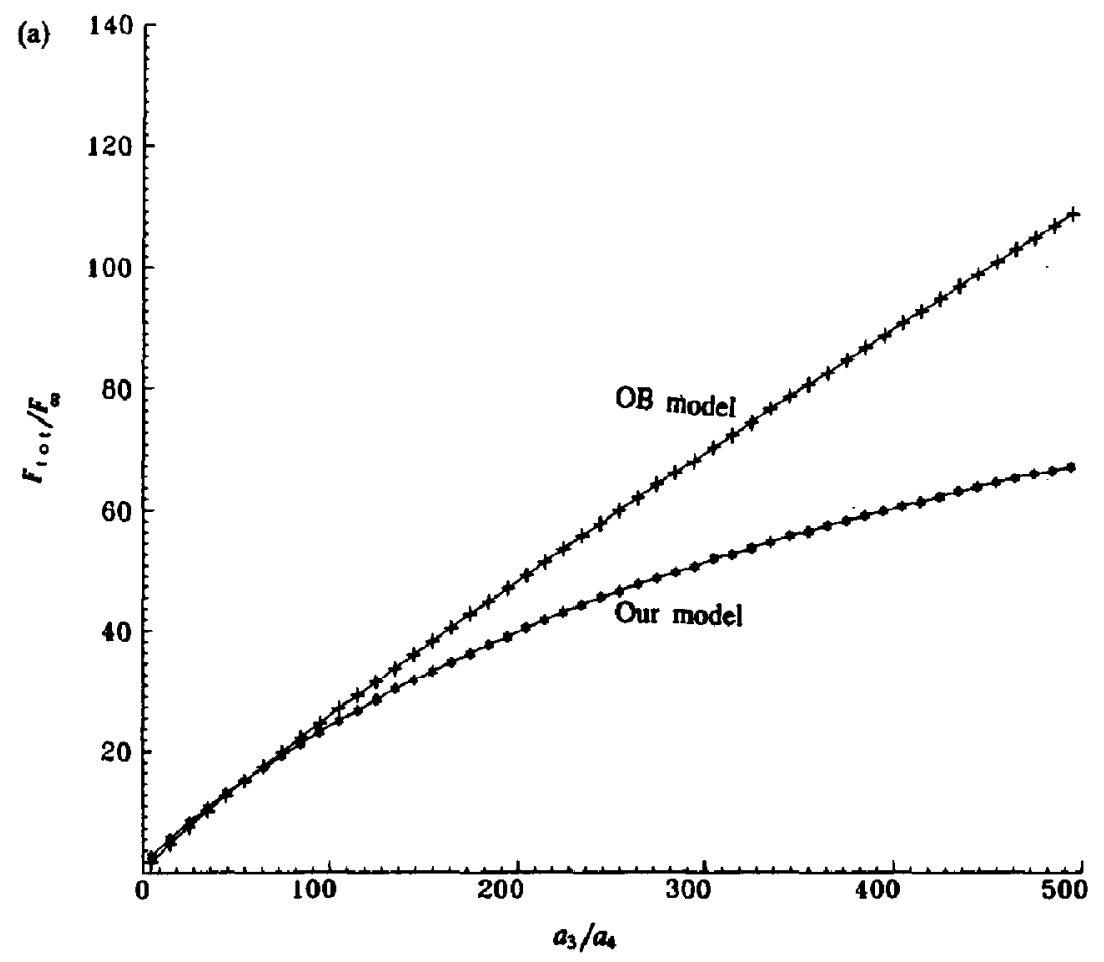

(b)

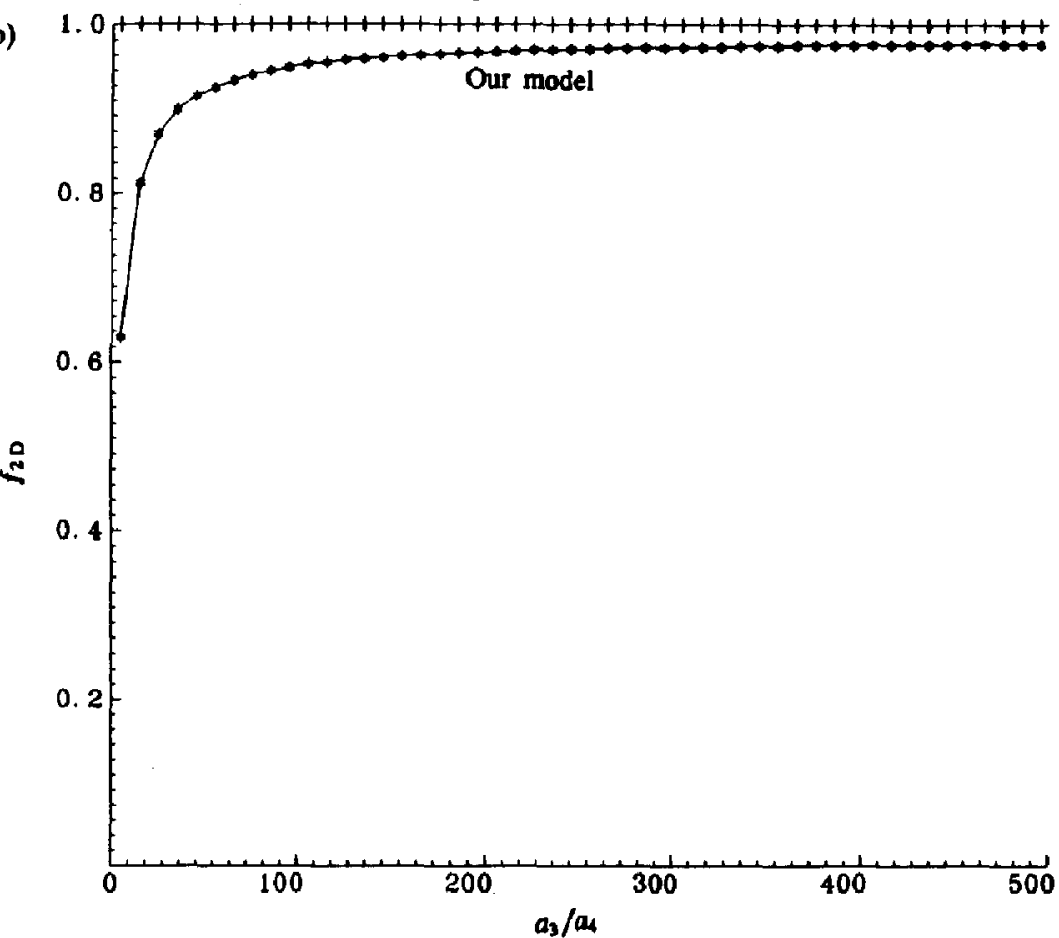

Fig. 11. A comparison of the present model with the Otto Berg $(O B)$ model at slow kinetic rates as a function of $a_{3} / a_{4}$ : (a) $F_{\text {tot }}$, and (b) $f_{2 \mathrm{D}}$. Parameters used: $a_{3}=10^{-3}$, and $a_{1}=a_{2}=500$. 

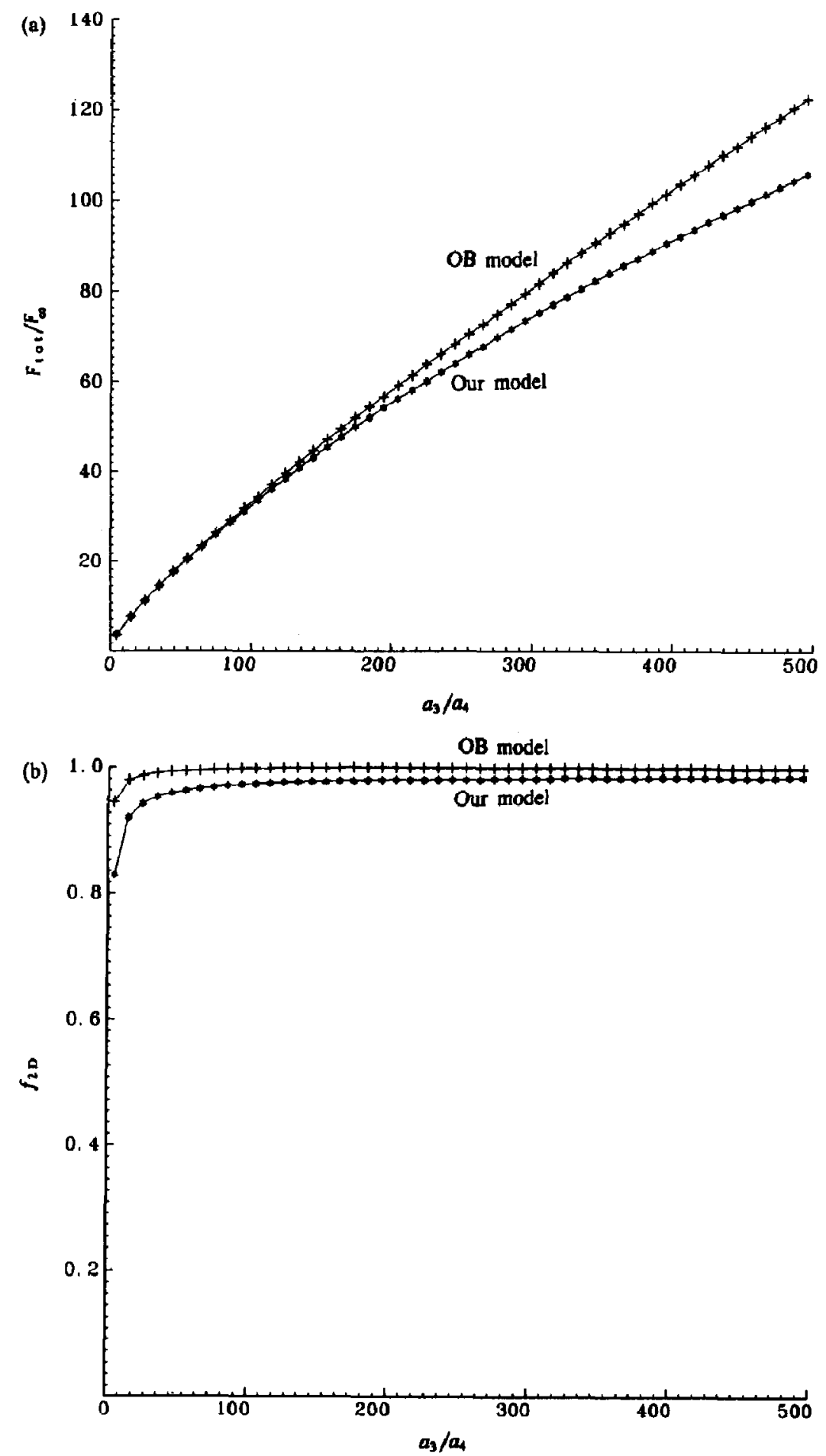

Fig. 12. A comparison of the present model with the OB model at fast kinetic rates as a function of $a_{3} / a_{4}:$ (a) $F_{\text {tot }}$, and (b) $f_{2 \mathrm{D}}$. Parameters used: $a_{3}=1$, and $a_{1}=a_{2}=500$. 
Figs. 11 and 12 show that the two models approach each other as the kinetic rates become faster. This is not surprising since the OB model is more appropriate for the limiting case of $a_{3}$ and $a_{4} \rightarrow \infty$, whereas our model does not have this constraint.

\section{Discussion and conclusions}

Ligands can be captured by a surface target either through direct bulk diffusion or surface diffusion following reversible adsorption to the surface. Given the presence of nonspecific adsorption, the ability of the adsorbed ligands to surface diffuse will always enhance reaction rates with a perfect sink target located in the surface in steady state. The questions addressed here are: (a) under what conditions does the enhancement become significant; and (b) how does the magnitude of the enhancement depend on the physical parameters?

Correct prediction of the relative contribution of the $2 \mathrm{D}$ and $3 \mathrm{D}$ pathways to the total rate of capture can help to elucidate the mechanism by which the target captures ligands. We have solved a steady state boundary value problem for a perfect sink disk target in the surface, taking into account bulk and surface diffusion coefficients $D$ and $D_{\mathrm{s}}$ and adsorption/desorption kinetic rate constants $k_{\mathrm{a}}$ and $k_{\mathrm{d}}$ at non-target regions. Solutions have been successfully found by numerical computation on a PC. The results show that the rate of capture from the surface depends non-linearly on $D_{\mathrm{s}}, K_{\mathrm{eq}}, k_{\mathrm{a}}, k_{\mathrm{d}}$ and geometrical dimensions. In particular, we demonstrate that not only is the non-target reqion equilibrium constant $K_{\text {eq }}$ important in determining the rate of capture from the surface, but so are the kinetic rate constants $k_{\mathrm{a}}$ and $k_{\mathrm{d}}$ separately. As is clear from Fig. 8, the dependence on the kinetic rate constants individually is quite strong for all situation in which the 2D flux is significant; in the ranges shown, there is no limit beyond which these kinetic rate constants individually are not important.

In all cases, the combination of surface adsorption and diffusion enhances the total rate of capture. The results should be useful for predict- ing $3 \mathrm{D}$ and $2 \mathrm{D}$ reaction rates of biological membrane bound receptor clusters and substrate-immobilized enzymes. The rate parameters that are needed for this prediction, the kinetic rate constants and surface diffusion coefficient, are all calculatable from measurements taken with available experimental techniques, notably by total internal reflection/fluorescence recovery after photobleaching [10-13].

The geometries usually chosen for simplicity in diffusional capture calculations-isolated or uniformly spaced sinks on a sphere with a ligand source at infinity or, as presented here, uniformly spaced targets on a plane with a ligand source at a finite distance $H$ above the plane-are clearly not "general". The particular geometrical assumptions do affect the absolute rates of capture. However, the most significant results in our calculations, the relative rates of $2 \mathrm{D}$ vs. $3 \mathrm{D}$ capture given by $f_{2 \mathrm{D}}$, are rather insensitive to changes in the intertarget distance $2 R_{2}$ and the ligand source height $H$ over a large range (e.g., for $a_{1}=R_{2} / R_{1}$ greater than 40 and for $a_{2}=H / R_{1}$ greater than 10 with the particular other parameters as chosen for Fig. 5d). Therefore, we would expect our approach for calculating $f_{2 \mathrm{D}}$ to be valid even if the sinks were spaced randomly (but sparsely) rather than uniformly, and if the surface were curved rather than flat. We would expect the approach to be less valid for randomly (but densely) distributed receptors where the average spacing between sinks is small, which for our parameters means $R_{2} / R_{1}<40$. Nevertheless, the approach is completely unrestricted with respect to choice of $H$; the figures assume $H \gg R_{1}$ only for casicr comparison with prcvious models.

The absolute rates are a more sensitive function of geometry than the relative rates. This is a general difficulty with calculations modeling chemoreception: an actual biological receptive surface is nowhere close to being either an isolated sphere in a ligand sea of infinite depth nor a plane in a finite sea. The calculations described here, which offer $a_{1}$ and $a_{2}$ as input parameters, show both the effects of varying the cylinder height and radius (e.g., Figs. 3 and 5) and the approximate range of geometrical parameters over which the exact geometry is not critical. 
The treatment here is "exact" in the sense that no limiting approximations were employed to solve the assumed model, and the results should be valid over a wide range of rate constants, surface target densities, and diffusion coefficients. However, the assumption that the target is a perfect sink is not necessarily appropriate for all cases of specific binding to a surface target. Presumably, if the sink is irreversible but "imperfect" in that not every collision leads to disappearance of the ligand, then the target still can be modeled as perfect sink but with a smaller radius. On the other hand, if binding to the target is reversible so that the ligand eventually returns unaltered to the surroundings (as does acetylcholine at its receptor), then the steady state solution describes an equilibrium situation with no net flows. In that case, no depletion zone exists in steady state and the theory contained herein is inappropriate. "Collision" rather than "capture" rates become relevant, and it is still likely that $2 \mathrm{D}$ diffusion significantly enhances the total rate of collision. However, since the ligands and receptors are in reversible equilibrium, the non-specific kinetic rate constants $k_{\mathrm{a}}$ and $k_{\mathrm{d}}$ will affect the degree of enhancement only through their ratio, the equilibrium constant $K_{\text {eq }}$.

Although a biological cell surface receptor is often modeled in the literature as a perfect sink, it may capture just one ligand and then the whole complex effectively disappears via internalization, migration to coated pits, etc, to be replaced on the average by another receptor at a random location. Of course, statistical local depletion zones of tiny amplitude do develop around the bound-up receptors, but the newly incorporated receptors appear elsewhere in general and, on the average, they do not sample those local depletion zones. Therefore, all new binding occurs in a statistically uniform concentration, and the theory herein is again inappropriate. However, a non-local 3D depletion zone may still develop over the whole surface, which acts as an imperfect but irreversible sink. The profile and depth of this nonlocal depletion zone, and the $2 \mathrm{D}$ diffusion enhancement of the overall rate of irreversible capture by the surface, may still be func- tions of $k_{\mathrm{a}}$ and $k_{\mathrm{d}}$ separately (not just through $K_{\text {eq }}$ ). The details of a solution to this problem remain to be worked out.

\section{Acknowledgements}

Special thanks to Dr. Robert M. Fulbright for his generous help and important suggestions on the theory and the programming. We also thank Dr. M.J. Weinstein for his helpful advice in trying to solve the problem analytically, Dr. P.L. Roe for his help in searching for numerical methods, and Dr. Nancy L. Thompson for many useful discussions on this topic. This project was supported by NIH 14565 and NSF DMB-8805296 to D.A.

\section{References}

1 G. Adam and M. Delbrück, in: Structural chemistry and molecular biology, eds. A. Rich and N. Davison (W.H. Freeman, San Francisco, CA, 1968) p. 198.

2 D. Axelrod, J. Membrane Biol. 75 (1983) 1.

3 H. Berg and E. Purcell, Biophys. J. 30 (1977) 193.

4 O. Berg, Biophys. J. 47 (1985) 1.

5 R.I. Cukier, J. Chem. Phys. 79 (1983) 2430.

6 A. Rosevear, J.F. Kennedy, and J.M.S. Cabral, Immobilized enzymes and cells (Hilger, Philadelphia, PA, 1987).

7 G. Venkatakrishnan, C.A. McKinnon, C.G. Pilapil, D.E. Wolf and A.H. Ross, Biochemistry 30 (1991) 2748.

8 M.A. McCloskey and M.-M. Poo, J. Cell Biol. 102 (1986) 88.

9 R. Schranner and P.H. Richter, Biophys. Chem. 8 (1978) 135 .

10 N. Thompson, T. Burghardt, and D. Axelrod, Biophys. J. 33 (1981) 435.

11 D. Axelrod, E.I. Hellen and R.M. Fulbright, in: Topics in fluorescence spectroscopy, Vol. 3: Biochemical applications, ed. J. Lakowicz (Plenum, New Yotk, NY, 1992), p. 289.

12 E.H. Hellen and D. Axelrod, J. Fluorescence 1 (1991) 113.

13 R.M. Fulbright and D. Axelrod, submitted.

14 J. Crank, The mathematics of diffusion, second edition (Oxford, New York, 1975) p. 42.

15 W.H. Press, B.P. Flannerry, S.A. Teukolsky, and W.T. Vetterling, Numerical recipes (Cambridge, New York, NY, 1989) Chap. 17.

16 D.A. Anderson, J.C. Tannehill, and R.H. Pletcher, Computational fluid mechanics and heat transfer (Hemisphere, New York, NY, 1984) p. 247. 